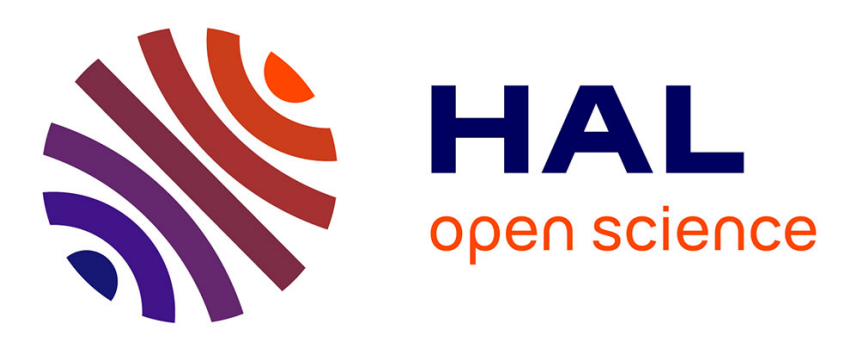

\title{
Analytic one-electron properties at the 4-component relativistic coupled cluster level with inclusion of spin-orbit coupling
}

Avijit Shee, Lucas Visscher, Trond Saue

\section{- To cite this version:}

Avijit Shee, Lucas Visscher, Trond Saue. Analytic one-electron properties at the 4-component relativistic coupled cluster level with inclusion of spin-orbit coupling. Journal of Chemical Physics, 2016, 145 (18), pp.184107. 10.1063/1.4966643 . hal-01430133

\section{HAL Id: hal-01430133 \\ https://hal.science/hal-01430133}

Submitted on 21 Jan 2020

HAL is a multi-disciplinary open access archive for the deposit and dissemination of scientific research documents, whether they are published or not. The documents may come from teaching and research institutions in France or abroad, or from public or private research centers.
L'archive ouverte pluridisciplinaire HAL, est destinée au dépôt et à la diffusion de documents scientifiques de niveau recherche, publiés ou non, émanant des établissements d'enseignement et de recherche français ou étrangers, des laboratoires publics ou privés. 


\section{AlP $\mid \begin{aligned} & \text { The Journal of } \\ & \text { Chemical Physics }\end{aligned}$}

Analytic one-electron properties at the 4-component relativistic coupled cluster level with inclusion of spin-orbit coupling

Avijit Shee, Lucas Visscher, and Trond Saue

Citation: The Journal of Chemical Physics 145, 184107 (2016); doi: 10.1063/1.4966643

View online: http://dx.doi.org/10.1063/1.4966643

View Table of Contents: http://scitation.aip.org/content/aip/journal/jcp/145/18?ver=pdfcov

Published by the AIP Publishing

\section{Articles you may be interested in}

Description of spin-orbit coupling in excited states with two-component methods based on approximate coupled-cluster theory

J. Chem. Phys. 142, 104109 (2015); 10.1063/1.4908536

Analytic second derivatives in closed-shell coupled-cluster theory with spin-orbit coupling

J. Chem. Phys. 131, 164113 (2009); 10.1063/1.3245954

Analytic energy gradients in closed-shell coupled-cluster theory with spin-orbit coupling

J. Chem. Phys. 129, 174110 (2008); 10.1063/1.3000010

High-order electron-correlation methods with scalar relativistic and spin-orbit corrections

J. Chem. Phys. 126, 024104 (2007); 10.1063/1.2423005

An ab initio two-component relativistic method including spin-orbit coupling using the regular approximation J. Chem. Phys. 113, 4052 (2000); 10.1063/1.1288387

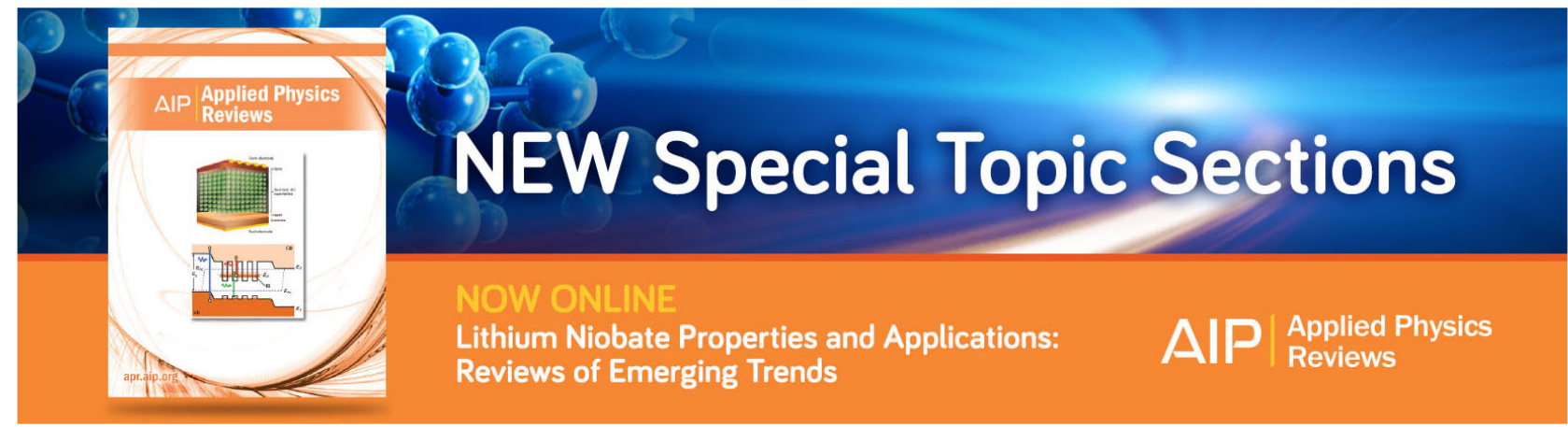




\title{
Analytic one-electron properties at the 4-component relativistic coupled cluster level with inclusion of spin-orbit coupling
}

\author{
Avijit Shee, ${ }^{1}$ Lucas Visscher, ${ }^{2}$ and Trond Saue ${ }^{1}$ \\ ${ }^{1}$ Laboratoire de Chimie et Physique Quantiques (UMR 5626), CNRS/Université Toulouse III - Paul Sabatier, \\ 118 Route de Narbonne, F-31062 Toulouse Cedex, France \\ ${ }^{2}$ Department of Theoretical Chemistry, Faculty of Sciences, Vrije Universiteit Amsterdam, De Boelelaan 1083, \\ 1081 HV Amsterdam, The Netherlands
}

(Received 12 June 2016; accepted 17 October 2016; published online 10 November 2016)

\begin{abstract}
We present a formulation and implementation of the calculation of (orbital-unrelaxed) expectation values at the 4-component relativistic coupled cluster level with spin-orbit coupling included from the start. The Lagrangian-based analytical energy derivative technique constitutes the basic theoretical framework of this work. The key algorithms for single reference relativistic coupled cluster have been implemented using routines for general tensor contractions of up to rank- 2 tensors in which the direct product decomposition scheme is employed to benefit from double group symmetry. As a sample application, we study the electric field gradient at the bismuth nucleus in the $\mathrm{BiX}(X=\mathrm{N}, \mathrm{P})$ series of molecules, where the effect of spin-orbit coupling is substantial. Our results clearly indicate that the current reference value for the nuclear quadrupole moment of ${ }^{209} \mathrm{Bi}$ needs revision. We also have applied our method to the calculation of the parity violating energy shift of chiral molecules. The latter property is strictly zero in the absence of spin-orbit coupling. For the $\mathrm{H}_{2} \mathrm{X}_{2}$ $(\mathrm{X}=\mathrm{O}, \mathrm{S}, \mathrm{Se}, \mathrm{Te})$ series of molecules the effect of correlation is found to be quite small. Published by AIP Publishing. [http://dx.doi.org/10.1063/1.4966643]
\end{abstract}

\section{INTRODUCTION}

Molecular properties are generally defined in terms of the response of a molecule to perturbations, such as electromagnetic fields or geometric displacements. The former are introduced via scalar and vector potentials into the electronic Hamiltonian through the principle of minimal electromagnetic coupling, ${ }^{1}$ which expresses a relativistic coupling of particles and fields. ${ }^{2}$ The resulting minimal substitution is employed both in the nonrelativistic and relativistic domains. This allows one, for instance, to study magnetic properties in a nonrelativistic framework, although one may argue that magnetic interactions, and in particular magnetic induction, vanish in the nonrelativistic limit. ${ }^{3}$

An important manifestation of magnetic induction is spinorbit coupling (SOC) ${ }^{4}$ which is often treated as a perturbation for efficiency reasons. However, SOC has a profound impact on various molecular properties and may thereby require a non-perturbative treatment. An example is the g-tensor of electron paramagnetic resonance (EPR) spectroscopy which would be strictly twice the $3 \times 3$ identity matrix at the nonrelativistic or scalar relativistic level ${ }^{5}$ and would not show the dependence on molecular electronic structure that makes EPR such a useful technique. Another example is the parity violation (PV) energy difference between enantiomers of chiral molecules which vanishes if SOC is ignored. ${ }^{6-8}$ The spin-orbit interaction may also have a sizable effect on NMR shieldings. ${ }^{9,10}$

The heavy elements, which show the most pronounced relativistic effects, also have many electrons, and electron correlation may therefore also have a significant impact on properties of molecules containing such elements. The electric field gradient (EFG) tensor at a nuclear position is an example; it has been shown for several systems that the correlation effect is almost equivalent to the total mean-field relativistic effect, ${ }^{11}$ while the contribution of SOC can be equally significant. ${ }^{12}$ This is due to the fact that this molecular property probes the electronic wavefunction both in the valence region and in the vicinity of nuclei. Other examples of properties that sensitively depend on the electronic wave function near the nuclei are the contact density, which has been used for the study of Mössbauer isomer shift, ${ }^{13}$ and hyperfine coupling constants. ${ }^{14}$ Like the EFG these properties can be used to study the chemical environment, as they primarily depend on the core tails of valence orbitals. The involvement of the valence region which is strongly influenced by electron correlation warrants a careful treatment of correlation.

The above considerations suggest that we in many situations need to consider scalar relativity, spin-orbit coupling, and electron correlation in a combined way. ${ }^{15}$ If we restrict ourselves to wavefunction theory, then the secondorder Møller-Plesset (MP2) gradient code, based on the 4component relativistic Dirac-Coulomb (DC) Hamiltonian, by van Stralen et al. ${ }^{16}$ is one of the few efforts in which both scalar and SOC relativistic effects are included from the outset. Technically easier is to only include scalar relativity in the mean field calculation and then introduce SOC in the subsequent correlation stage. Such a two-step procedure is more efficient in a coupled cluster (CC) framework than a Configuration Interaction (CI) one, since the former method is more efficient at recovering spin-orbit polarization. ${ }^{17}$ Wang et al. ${ }^{18}$ have reported a two-step procedure where an effective 
one-electron spin-orbit operator is added at the CC level following a scalar relativistic Hartree-Fock (HF) calculation. An analytic gradient formulation and implementation of that approach has been reported by Wang and Gauss. ${ }^{19}$ An alternative approach is to perform scalar relativistic CC energy calculations and then add SOC as an additional perturbation in response theory. Cheng and Gauss have accordingly recently reported $\mathrm{CC}$ analytic gradients based on the 4-component spinfree (SF) DC Hamiltonian ${ }^{20}$ or the spinfree exact 2-component relativistic Hamiltonian (SF-1sX2C). ${ }^{21,22}$ However, for heavy elements the valence electronic structure may be qualitatively altered by SOC and a response theory treatment will need to go through higher orders and can even fail dramatically in a valence-only treatment of electron correlation. $^{23}$

Until now, where the DC coupled cluster based property calculations were deemed to be necessary, a numerical differentiation route was taken. ${ }^{13,24,25}$ However, finite-field calculations are plagued by higher computational cost, are more sensitive to numerical noise, and cannot be straightforwardly extended to time-dependent properties. They are, therefore, better replaced by an analytical implementation. The major goal of this work is to provide a fully DC Hamiltonian based CC analytic gradient code that allows us to capture all the essential physics in a consistent way.

The formulation we have used is largely inspired by corresponding nonrelativistic theories. However, due to the very nature of the Dirac equation there are some differences, namely, in the treatment of symmetry and the nature of the orbital relaxation. They will be clarified in Section II. The method has been implemented in the DIRAC quantum chemistry package. ${ }^{26}$ All the pertinent details of this implementation will be discussed in Section III. Sample applications of the present method are reported in Section IV. Finally, a summary of our work as well as future prospects will be given in Section V.

\section{THEORY}

Coupled cluster theory is (in practice) a non-variational theory based on a non-unitary exponential parametrization of the wave function

$$
|C C\rangle=\exp (\hat{T})\left|\Phi_{0}\right\rangle, \quad \hat{T}=\sum_{l=1} t_{l} \hat{\tau}_{l},
$$

where $t_{l}$ and $\hat{\tau}_{l}$ denote cluster amplitudes and excitation operators, respectively. The reference $\Phi_{0}$ typically, and in this work, refers to the Hartree-Fock (HF) determinant. Furthermore, in the present work $\hat{T}$ is restricted to single and double excitations,

$$
\hat{T}=\hat{T}_{1}+\hat{T}_{2}, \quad \hat{T}_{1}=\sum_{i a} t_{i}^{a} a_{a}^{\dagger} a_{i}, \quad \hat{T}_{2}=\frac{1}{4} \sum_{i j a b} t_{i j}^{a b} a_{a}^{\dagger} a_{b}^{\dagger} a_{j} a_{i} .
$$

Here and in the following indices $i, j, \ldots, n, o$ refer to occupied (hole) orbitals, indices $a, b, \ldots, f, g$ refer to virtual (particle) orbitals, and $p, q, r, s$ are general orbital indices. A troublesome aspect of 4-component relativistic theory is the presence of negative-energy orbitals which in turn implies that the electronic Hamiltonian, here taken as the Dirac-Coulomb Hamiltonian (see below), has no bound solutions. ${ }^{27}$ A solution is provided by the no-pair approximation, ${ }^{28,29}$ in which the electronic Hamiltonian is embedded by operators projecting out negative-energy orbitals. In practice, molecular orbitals $\left\{\varphi_{p}\right\}$ are optimized at the HF level and then the negativeenergy orbitals are eliminated from the correlated level. The present work is based on this no-pair approximation, hence, unless otherwise stated, orbital indices in the following refer exclusively to positive-energy orbitals.

Equations for the energy and the cluster amplitudes are conveniently given in terms of the similarity-transformed Hamiltonian $\hat{H}=\exp (-\hat{T}), \hat{H} \exp (\hat{T})$,

$$
\begin{aligned}
& \left\langle\Phi_{0}|\hat{\bar{H}}| \Phi_{0}\right\rangle=E, \\
& \left\langle\Phi_{l}|\hat{\bar{H}}| \Phi_{0}\right\rangle=0, \quad\left|\Phi_{l}\right\rangle=\hat{\tau}_{l}\left|\Phi_{0}\right\rangle .
\end{aligned}
$$

We now consider the case where the electronic Hamiltonian is extended by perturbations, each characterized by a perturbation strength $\varepsilon_{X}$, collected in the vector $\varepsilon$,

$$
\hat{H}=\hat{H}_{0}+\sum_{X} \varepsilon_{X} \hat{H}_{X}
$$

The zeroth-order Hamiltonian will be the 4-component relativistic Dirac-Coulomb Hamiltonian. It has the same generic form,

$$
\hat{H}_{0}=V_{N N}+\sum_{i} \hat{h}(i)+\frac{1}{2} \sum_{i \neq j} \hat{g}(i, j),
$$

as the nonrelativistic electronic Hamiltonian, including the electrostatic repulsion $V_{N N}$ of clamped nuclei, but the oneelectron operator $\hat{h}$ is the Dirac Hamiltonian,

$$
\hat{h}=\left[\begin{array}{ll}
V_{e N} & c(\boldsymbol{\sigma} \cdot \mathbf{p}) \\
c(\boldsymbol{\sigma} \cdot \mathbf{p}) & V_{e N}-2 m c^{2}
\end{array}\right],
$$

where $c$ is the speed of light ( $c=137.0359998$ a.u.). The electron-nucleus interaction $V_{e N}$ is expressed in terms of the scalar potential $\phi_{A}$ of each nucleus $A$,

$V_{e N}=-e \sum_{A} \phi_{A}, \quad \phi_{A}\left(\mathbf{r}_{1}\right)=\frac{1}{4 \pi \varepsilon_{0}} \int \frac{\rho_{A}\left(\mathbf{r}_{2}\right)}{\left|\mathbf{r}_{1}-\mathbf{r}_{2}\right|} d^{3} \mathbf{r}_{2}$,

where we generally employ a Gaussian model for the nuclear charge distribution. ${ }^{30}$ The electron-electron interaction $\hat{g}$ is the instantaneous Coulomb interaction which is the zeroth-order term in a perturbational expansion in $c^{-2}$ of the fully relativistic interaction in Coulomb gauge.

Molecular properties may be defined in terms of perturbation (Maclaurin) expansions of expectation values in the framework of response theory. ${ }^{31-35}$ For fully variational methods, static molecular properties are conveniently expressed as derivatives of the energy with respect to perturbational strengths at zero field $(\boldsymbol{\varepsilon}=\mathbf{0})$, where the connection to expectation values is assured by the HellmannFeynman theorem. Simplifications are then obtained due to Wigner's $2 n+1$ rule. $^{36,37}$ Since CC theory is non-variational 
we instead consider the Lagrangian ${ }^{33,38}$

$$
\begin{aligned}
L^{\mathrm{CC}}(\boldsymbol{\varepsilon}, \mathbf{t}, \boldsymbol{\lambda}, \boldsymbol{\kappa}, \overline{\boldsymbol{\kappa}})= & \left\langle\Phi_{0}|(1+\Lambda) \hat{\bar{H}}| \Phi_{0}\right\rangle \\
& +\sum_{a i}\left(\bar{\kappa}_{a i} \frac{\partial E^{H F}}{\partial \kappa_{a i}}+\bar{\kappa}_{a i}^{*} \frac{\partial E^{H F}}{\partial \kappa_{a i}^{*}}\right)
\end{aligned}
$$

where $\Lambda$ comprise the Lagrangian multipliers for the CC amplitude equations (Eq. (4)), for which a $2 n+2$ rule holds. ${ }^{39-41}$ The second term represents orbital relaxation and the virtual index $a$ here refers to virtual orbitals of both positive and negative energies. We assume the use of a unitary exponential parametrization of the HF trial function, which assures orthonormality of orbitals throughout optimization. ${ }^{4-45} \kappa_{a i}$ and $\bar{\kappa}_{a i}$ refer to the orbital rotation parameters and the corresponding Lagrange multipliers, respectively. These are elements of anti-Hermitian and Hermitian matrices, respectively, and one may note that only the virtual-occupied blocks are included since all other elements are redundant. ${ }^{44}$ In the 4-component relativistic domain the orbital rotation parameters naturally split into two classes: parameters $\left\{\kappa_{a i}^{++}\right\}$, involving rotations between the positive-energy occupied and virtual orbitals, and $\left\{\kappa_{a i}^{-+}\right\}$, corresponding to rotations between positive-energy occupied orbitals and negative-energy virtual orbitals. The HF energy is minimized with respect to the former set, but maximized with respect to the latter, in accordance with the minmax principle of Talman. ${ }^{46,47}$ This corresponds to the implicit use of projection operators embedding the electronic Hamiltonian and reflects the use of the no-pair approximation. ${ }^{27,28}$

In the above approach the redundant orbital rotations are set to zero. This corresponds to an algorithm of least change, ${ }^{48,49}$ only assuring that the occupied-virtual blocks of the Fock matrix are zero. For an explicit separation of orbitals of positive and negative energy one may impose canonical orbitals at all perturbation strengths. This can be realized by extension of the CC Lagrangian, Eq. (9),

$$
L^{\mathrm{CC}} \rightarrow L^{\mathrm{CC}}+\sum_{p q} \zeta_{p q}\left(F_{p q}-\varepsilon_{p} \delta_{p q}\right),
$$

where $\left\{\zeta_{p q}\right\}$ constitutes a new set of Lagrange multipliers. ${ }^{40,50}$ In this approach all orbital rotations come into play, including rotations within orbital classes. The two approaches therefore generally give different results when some orbitals are selected as inactive, of which the no-pair approximation is a special case. The canonical approach has the advantage of adhering closer to the usual picture of the Dirac sea, whereas the "minimal" approach allows the extension of the no-pair approximation to situations where orbital energies are not available. For instance, 4-component relativistic MCSCF calculations use second-order optimization based on the Talman minmax principle, ${ }^{51,52}$ and allow the complete relaxation of the no-pair projection operators at the correlated level. ${ }^{29,47} \mathrm{We}$ intend to explore and compare these two approaches in future work.

It may be noted that the Lagrangian formalism can also be used in the case of perturbation-dependent orbitals, as will be the case in geometry optimization or the use of London orbitals ${ }^{53,54}$ (also known as gauge-including atomic orbitals (GIAOs)), ${ }^{55}$ provided a suitable orbital connection ${ }^{56}$ is employed to guarantee orthonormality. However, upon taking derivatives of the Lagrangian, Eq. (9), with respect to perturbations, as we do in the following, further contributions will appear. In the present contribution we therefore limit attention to perturbation-independent orbitals.

Since coupled cluster theory is very robust and usually already provides adequate orbital relaxation through the $T_{1}$ cluster amplitudes ${ }^{57}$ it is reasonable to ignore it, as we do in the present work. An exception is the calculation of magnetic properties for which the negative-energy (NE) orbitals, which are excluded from the excitation manifold, are essential to get the diamagnetic contribution to magnetic properties. ${ }^{58,59}$ For other molecular properties the inclusion of orbital relaxation at the CC level is a matter of debate-for some static first order properties it certainly shows improvement, ${ }^{60,61}$ and for others it is not recommended, ${ }^{62}$ while for dynamic properties problems arise due to the inaccurate Hartree-Fock poles. ${ }^{35}$ For calculations of heavy elements there is the additional complication of the relaxation of core orbitals that are usually left uncorrelated. We plan to come back to these issues in future work. As of now we will work with the reduced Lagrangian appropriate for orbital-unrelaxed calculations,

$$
L(\boldsymbol{\varepsilon}, \mathbf{t}, \boldsymbol{\lambda})=\left\langle\Phi_{0}|\hat{\bar{H}}| \Phi_{0}\right\rangle+\sum_{l=1} \lambda_{l}\left\langle\Phi_{l}|\hat{\bar{H}}| \Phi_{0}\right\rangle,
$$

where we have used a linear parametrization of the Lagrangian multiplier, that is,

$$
\Lambda=\sum_{l} \lambda_{l} \hat{\tau}_{l}^{\dagger}
$$

where "l" indicates the rank of different excited states. Truncation of the $\Lambda$ operator is dictated by the rank of the T operators. For the coupled-cluster singles-and-doubles (CCSD) analytic gradient method considered in this work, we therefore take up to doubly de-excitation type $\Lambda$ s. In passing, we may note that the appropriate Lagrangian for the calculation of orbital-unrelaxed expectation values at the 4-component relativistic MP2 level is obtained from the above Lagrangian by replacing the similarity-transformed Hamiltonian $\hat{\hat{H}}$ in the first and second term by its secondand first-order component, respectively (assuming optimized orbitals). ${ }^{40,50,63}$

We apply two stationary conditions on the Lagrangian in Equation (11),

$$
\begin{aligned}
& \frac{\partial L}{\partial \mathbf{t}}=\mathbf{0} \\
& \frac{\partial L}{\partial \boldsymbol{\lambda}}=\mathbf{0} .
\end{aligned}
$$

Equation (14) is the already implemented equation to determine the T-amplitudes, whereas Equation (13) defines the $\Lambda$-equation used to determine the Lagrange multipliers. Equations (13) and (14) can also be interpreted as equations for the right and left eigenvectors, respectively, of the nonHermitian similarity-transformed Hamiltonian, ${ }^{64,65}$

$$
\begin{array}{ll}
(\hat{\bar{H}}-E)\left|\Psi_{R}\right\rangle=0, & \left|\Psi_{R}\right\rangle=\left|\Phi_{0}\right\rangle, \\
\left\langle\Psi_{L}\right|(\hat{\bar{H}}-E)=0, & \left\langle\Psi_{L}\right|=\left\langle\Phi_{0}\right|(1+\Lambda) .
\end{array}
$$


This means that the $\Lambda$-equation, Equation (13), defines a dual vector of the coupled cluster ket vector, which enables the calculation of molecular properties as an expectation value. In fact, using the stationary conditions, Eqs. (13) and (14), we obtain

$$
\left.\frac{d L}{d \varepsilon_{X}}\right|_{\varepsilon=\mathbf{0}}=\left.\frac{\partial L}{\partial \varepsilon_{X}}\right|_{\varepsilon=\mathbf{0}}=\left\langle\Psi_{L}\left|\hat{\bar{H}}_{X}\right| \Psi_{R}\right\rangle,
$$

which corresponds to the expectation value of the operator $\hat{H}_{X}$. Equation (16) thereby constitutes a generalized HellmannFeynman theorem. Defining CC density matrices

$$
\begin{aligned}
\gamma_{p}^{q} & =\left\langle\Psi_{L}\left|\exp (-\hat{T}) a_{q}^{\dagger} a_{p} \exp (\hat{T})\right| \Psi_{R}\right\rangle, \\
\Gamma_{p q}^{r s} & =\left\langle\Psi_{L}\left|\exp (-\hat{T}) a_{r}^{\dagger} a_{s}^{\dagger} a_{q} a_{p} \exp (\hat{T})\right| \Psi_{R}\right\rangle,
\end{aligned}
$$

the expectation value can be expressed as a trace between the density and the operator matrix elements,

$$
\left\langle\hat{H}_{X}\right\rangle=\sum_{p q} \gamma_{p}^{q} h_{X ; p q}
$$

All the properties we will be dealing with in the present work are of this expectation value type. The explicit working equations for intermediates and $\Lambda$ amplitude equations, as well as density matrices, are given in Appendix A.

The size-extensivity of the evaluated properties follows from the following argument: Taking the derivative of the Lagrangian with respect to the amplitudes $\mathbf{t}$ in Equation (13) means from a set of closed terms constructed by $\mathrm{H}, \exp (\hat{T})$, and $\Lambda$, we take out the amplitude part of $\hat{T}$. The operator part of $\hat{T}$ acts on the ket side of the Lagrangian to generate an excited function which is then connected to the bra side via the de-excitation operators contained in $\Lambda$ (see Equation (12)). The stationarity condition tells us that the final result will be zero. The total $\Lambda$-equation may be written in the following algebraic form: ${ }^{66}$

$$
\begin{aligned}
\frac{\partial L}{\partial t_{m}}= & \left\langle\Phi_{0}|\hat{\bar{H}}| \Phi_{m}\right\rangle+\left\langle\Phi_{0}|[\Lambda, \hat{\bar{H}}]| \Phi_{m}\right\rangle \\
& +\sum_{k \neq 0}\left\langle\Phi_{0}|\hat{\bar{H}}| \Phi_{k}\right\rangle\left\langle\Phi_{k}|\Lambda| \Phi_{m}\right\rangle=0,
\end{aligned}
$$

where the sum over the complete excitation manifold in the final term is limited by the de-excitations contained in $\Lambda$. The above equation can be analyzed by diagrammatic techniques ${ }^{67,68}$ which reveals a connected quantity ${ }^{63,69} \hat{\bar{H}}$, an explicit connected quantity between $\hat{\bar{H}}$ and $\Lambda$, and a third disconnected term containing $\Lambda$ and $\hat{H}$. The evaluated $\Lambda \mathrm{s}$ are therefore not connected quantities. However, if we write Equation (16) more explicitly as follows

$$
\frac{\partial L}{\partial \epsilon_{X}}=\left\langle\Phi_{0}\left|\left[\Lambda, \hat{\bar{H}}_{X}\right]+\hat{\bar{H}}_{X}\right| \Phi_{0}\right\rangle
$$

we see that it contains an explicitly connected quantity between $\Lambda$ and $\hat{\bar{H}}_{X}$, where $\hat{\bar{H}}_{X}$ is a connected quantity since our converged $\mathrm{T}$ amplitudes are connected. Now the completely contracted nature of Equation (21) ensures that only the connected part of $\Lambda$ can contribute to the final property value, which in turn ascertains the size-extensivity of the evaluated properties.

\section{IMPLEMENTATION}

The RELCCSD module in DIRAC ${ }^{26}$ provides 2- and 4-component relativistic CCSD, CCSD(T), and Fock-space coupled cluster methods. ${ }^{70-72}$ In the present work, we have extended this module in such a way that it can handle any arbitrary tensor contraction. Any implementation along this line generally hinges on an efficient tensor contraction routine where the tensor can be of arbitrary rank. Recently, there has been considerable focus on the development of general purpose tensor contraction routines, such as the "Tensor Contraction Engine, ${ }^{, 73}$ Cyclops, ${ }^{74}$ SIAL,${ }^{75}$ libtensor,${ }^{76}$ and TiledArray ${ }^{77}$ In this work, our target is to achieve almost the same flexibility without yet focusing on the parallel efficiency of the algorithm. By flexibility is meant that we want to employ tensors of arbitrary operator structure and rank. It will, in turn, facilitate the implementation of the methods pertaining to both energy and molecular properties at the Single Reference Coupled Cluster (SRCC) level.

\section{A. General structure of the implementation}

The pivotal operation in the CC family of methods is the binary tensor contraction,

$$
A_{i j . .}^{k^{\prime} l^{\prime} .} * B_{k l . .}^{i^{\prime} j^{\prime} . .}=C_{i j \ldots k l . .}^{k^{\prime} l^{\prime} \ldots i^{\prime} j^{\prime} . .} .
$$

Following the Einstein summation convention whenever the upper primed (bra) and lower unprimed (ket) indices are the same, they define a contraction. For each contraction, the rank of the product tensor is reduced by 1 . All tensors - A, $\mathrm{B}$, and $\mathrm{C}-$ are anti-symmetrized tensors of arbitrary rank.

There are no readily available library routines which are able to perform such tensor operations. However, one can take advantage of Basic Linear Algebra Subroutines $(\mathrm{BLAS})^{78}$ by conveniently mapping a tensor contraction onto a matrix-matrix multiplication,

$A($ free, contractable $) * B($ contractable, free $) \rightarrow C($ free, free $)$.

In the present work we have generalized binary tensor contractions by "contraction_xyz" FORTRAN 90+ subroutines, where $x$ and $y$ are the ranks of the left and right input tensors, respectively, and $z$ is the rank of the output tensor. For the theories which incorporate up to singles and doubles excitation operators, we have written the subroutines corresponding to contraction classes $x y z=222$, $242,422,444,244$, and 424 to be able to carry out every possible tensor contraction. These routines identify the permutational and double group symmetry built into a tensor and preserve the optimal symmetry structure after the contraction. Reshaping of the tensors is implicit in these routines from the placement of indices. Furthermore, we need to provide information only from a diagrammatic expression, therefore they can easily be coupled with an automatic expression generator. We illustrate the use of these routines in Appendix B. 


\section{B. Sparsity and blocking of tensors by using double group symmetry}

Use of molecular symmetry provides dense and blocked structure to the tensors used in a coupled cluster contraction. This has been exploited most efficiently by Stanton et al. ${ }^{79}$ in their Direct Product Decomposition (DPD) scheme for the spatial symmetry groups.

For the relativistic molecular Hamiltonian we cannot work with simple spatial point group symmetry due to spinorbit coupling, which couples the spin and spatial degrees of freedom. Rather we shall work with the corresponding double groups, obtained by the introduction of a rotation $2 \pi$ about an arbitrary axis. ${ }^{80}$ These groups contain more irreps than the regular single group, but not necessarily twice the number. The extra irreps are called fermion irreps and the pre-existing ones boson irreps. Spinors span fermion irreps, whereas spinor products, operators, and integrands span boson irreps.

In the present work, we use double groups of $\mathrm{D}_{2 h}$ and its subgroups. However, not all of them are Abelian since the direct product between the $2 \pi$ rotation and other elements of the point group may generate elements which belong to the same class as some other element. In that case, we consider the highest Abelian subgroup of that double group. For example, the $\mathrm{D}_{2 h}^{*}$ double group contains one two-dimensional irrep, therefore we work with the Abelian subgroup $\mathrm{C}_{2 h}^{*}$ instead. For linear systems we have exploited the advantage of going beyond $\mathrm{D}_{2 h}^{*}$ symmetry by using double groups $C_{8 h}^{*}$ and $C_{16}^{*}$ for molecules with and without inversion symmetry, respectively. For the spin-free cases, we use the direct product group between the point group and the SU(2) group. These extensions provide very significant computational savings in realistic applications. This Abelian double group scheme, which is analogous to DPD, builds on the symmetry handling in the CCSD implementation by Visscher et al. ${ }^{71}$ In Appendix $\mathrm{C}$ we present that scheme in a bit more generalized perspective while recapitulating the essential details from that work.

Further symmetry reductions are possible by exploiting time reversal symmetry. ${ }^{70}$ However, in the present code Kramers-restriction is not imposed at the CC level. On the other hand, presently the initial HF calculations are carried out using a quaternion symmetry scheme ${ }^{81}$ that exploits both spatial and time reversal symmetry such that the input orbitals for the CC module form a Kramers basis. As a consequence, the input one- and two-electron integrals are real for certain point groups, a feature that is exploited in our calculations by defining an xGEMM subroutine that wraps around either the DGEMM or ZGEMM BLAS primitives, depending on the algebra of the point group (real or complex).

\section{Construction of expectation values}

The steps involved in a coupled cluster gradient calculation can be written down succinctly as follows:

1. iterative $\mathrm{HF}$ solution to get the MO basis;

2. transforming integrals in $\mathrm{AO}$ basis to the $\mathrm{MO}$ basis;

3. iterative solution of the CCSD amplitude equation to get the $\mathrm{T}_{1}$ and $\mathrm{T}_{2}$ amplitudes;
4. construction of the fixed intermediates, Eqs. (A4)-(A14), using $\mathrm{V}, \mathrm{F}, \mathrm{T}_{1}$ and $\mathrm{T}_{2}$;

5. iterative solution of the $\Lambda$-equations, Eqs. (A1) and (A2);

6. construction of one-particle density matrix, Eq. (A17), at the CC level with the help of converged $\mathrm{T}$ - and $\Lambda$ amplitudes;

7. back-transformation of the $\mathrm{CC}$ density matrix from the MO basis to the AO basis;

8. tracing the density matrix with the AO derivative integrals to get the desired property values.

Steps (4) and (5) use the contraction scheme described in the Section III A. Some intermediates involve integrals over four virtual orbital indices (VVVV). Because of the very high memory scaling of those terms, we have implemented them in a distributed fashion. The RELCCSD module in DIRAC distributes the VOVV (three virtual and one occupied) and VVVV type integrals on several nodes. In the present work, we gather all the distributed VOVV integrals on each node and continue with the distributed VVVV integrals. The intermediate which contains four virtual orbitals, that is, $W_{a b}^{e f}$ in Equation (A11) is calculated only with the locally available integrals on each node. Furthermore, when those intermediates are used in Equations (A10) and (A2) we use only the local contribution on each node. At the end we synchronise the $\delta_{a b}^{i j}$ and $W_{a m}^{e f}$ arrays to the master.

Step (6) in the above scheme needs careful consideration since the AO property matrices are generated in the framework of the quaternion symmetry scheme ${ }^{81}$ operating at the SCF level in the DIRAC program. Presently the input MOs to the CC module form a Kramer's basis, such that the 1-particle CC density matrix $\left({ }^{M O} \gamma\right)$ is expressed in terms of Kramer's pairs,

$$
\left(\begin{array}{ll}
A & B \\
C & D
\end{array}\right), \quad \begin{array}{ll}
A_{p q}=\gamma_{p q}, & B_{p q}=\gamma_{p \bar{q}}, \\
C_{p q}=\gamma_{\bar{p} q}, & D_{p q}=\gamma_{\overline{p q}} .
\end{array}
$$

In the closed-shell case, this matrix has a time-symmetric structure

$$
\left(\begin{array}{cc}
A & B \\
-B^{\star} & A^{\star}
\end{array}\right)
$$

and can be block-diagonalized by quaternion unitary transformation $^{81}$

$$
U=\left(\begin{array}{cc}
\mathbb{I} & \check{j} \mathbb{I} \\
\check{j} \mathbb{I} & \mathbb{I}
\end{array}\right),
$$

where $\mathbb{I}$ is the $2 \times 2$ unit matrix. One then proceeds with the upper block of the quaternion block-diagonalized matrix, with elements

$$
Q_{\gamma_{P Q}}=\operatorname{Re}\left(\gamma_{p q}\right)+\check{i} \operatorname{Im}\left(\gamma_{p q}\right)+\check{j} \operatorname{Re}\left(\gamma_{p \bar{q}}\right)+\check{k} \operatorname{Im}\left(\gamma_{p \bar{q}}\right) .
$$

Based on spatial symmetry the matrix may be further compressed to complex or real form.

The ${ }^{Q_{\gamma}}$ density matrix is next back-transformed to the AO basis

$$
Q_{\gamma_{\mu \nu}}=\sum_{P Q} Q_{c_{\mu P} Q_{\gamma_{P Q}}{ }^{*} c_{\nu Q}^{*}}
$$

In the final step the resulting $\mathrm{AO} \mathrm{CC}$ density matrix is traced with the appropriate $\mathrm{AO}$ property matrix. 
As mentioned the RELCCSD module of DIRAC does not exploit time reversal symmetry and can also be invoked to handle a single open shell (one unpaired electron). In that case the CC density matrix can be separated into a time symmetric and a time antisymmetric part, the latter allowing the calculation of expectation values of time antisymmetric operators such as the magnetic dipole or the hyperfine operator. This will be exploited in future work.

\section{SAMPLE APPLICATIONS}

In this section we will show two pilot applications of the DC-CCSD analytic gradient code. We first consider parity violation (PV) of chiral molecules which, as already mentioned, is a property that is strictly zero in the absence of SOC and for which we would like to benchmark a currently employed computational protocol based on density functional theory (DFT). ${ }^{82}$ Presently we have studied PV in the $\mathrm{H}_{2} \mathrm{X}_{2}(\mathrm{X}=\mathrm{O}, \mathrm{S}, \mathrm{Se}, \mathrm{Te})$ series of molecules. For the first two members of that series orbital-relaxed finite-field 4-component relativistic CC calculations are available. ${ }^{83} \mathrm{We}$ next consider the electric field gradient (EFG) at nuclear positions in the BiN and $\mathrm{BiP}$ diatomic molecules, which were already studied by Teodoro and Haiduke ${ }^{84}$ using a finite-field $\mathrm{CC}$ approach.

\section{A. Parity violation}

Upon extension of the conventional electromagnetic formulation of quantum chemistry to the electroweak regime a minute energy difference,

$$
\Delta E_{P V}=E_{P V}(L)-E_{P V}(R)=2 E_{P V}(L),
$$

is induced between the left (L)- and right (R)-handed enantiomers of chiral molecules. ${ }^{6,885,86}$ At the 4-component relativistic level the iterapairs violating (PV) energy $E_{P V}$ can be calculated as an expectation value of the nuclear spin-independent P-odd operator ${ }^{87}$

$H_{P V}=\sum_{A} H_{P V ; A}, \quad H_{P V ; A}=\frac{G_{F}}{2 \sqrt{2}} Q_{W, A} \sum_{i} \gamma_{i}^{5} \rho_{A}\left(r_{i}\right)$,

where $G_{F}=1.16637 \times 10^{-11} \quad \mathrm{MeV}^{-2}=2.22255 \times 10^{-14}$ $E_{h} a_{0}^{3}$ is the Fermi coupling constant. The summation runs over the nuclei, A, and electrons, i. The weak charge $Q_{W, A}=-N_{A}+Z_{A}\left(1-4 \sin ^{2} \theta_{w}\right)$, where $N_{A}$ and $Z_{A}$ is the number of neutrons and protons on each nucleus. $\theta_{w}$ is the Weinberg mixing angle; we have chosen a fixed value of 0.2319 for $\sin ^{2} \theta_{w}$. $\rho_{A}$ is the normalized nucleon density. $\gamma^{5}$ is the $4 \times 4$ chirality operator, which is given by

$$
\gamma^{5}=\left(\begin{array}{ll}
\mathbb{O} & \mathbb{I} \\
\mathbb{I} & \mathbb{O}
\end{array}\right) .
$$

The nuclear spin-dependent PV contribution has been neglected since it is zero for a closed-shell system. ${ }^{87}$

There is at present no experimental observation of the effect of parity violation in chiral molecules, but several experiments are in preparation. ${ }^{7,88-90}$ The present role of theory is to guide experiment towards suitable candidate molecules, but a successful observation would call for a direct confrontation between theory and experiment and allow to probe the standard model of the universe in the low-energy regime. ${ }^{91}$ Most present-day molecular PV calculations are carried out using DFT. As a first step towards benchmarking of this computational protocol we have investigated the $\mathrm{H}_{2} \mathrm{X}_{2}(\mathrm{X}=\mathrm{O}, \mathrm{S}, \mathrm{Se}, \mathrm{Te})$ series of molecules using our newly implemented $\mathrm{CC}$ gradient code. These are not ideal candidate molecules for actual experiments, but have been widely employed for calibration and analysis. ${ }^{8,83,87,92-107}$ In our study with the first candidate of that series, that is, $\mathrm{H}_{2} \mathrm{O}_{2}$, the $\mathrm{O}-\mathrm{O}$, and $\mathrm{H}-\mathrm{O}$ bond lengths as well as the $\mathrm{O}-\mathrm{O}-\mathrm{H}$ angles have been kept fixed, and the dihedral angle $\tau_{e}$ has been varied from $0^{\circ}$ to $180^{\circ}$ with a grid of $20^{\circ}$ to generate an $E_{P V}$ vs $\tau_{e}$ curve. The fixed structure parameters for $\mathrm{H}_{2} \mathrm{O}_{2}$ and the heavier homologues have been taken from the article by Laerdahl and Schwerdtfeger. ${ }^{87}$ We have considered a series of basis sets to estimate the minimal size which provides good accuracy. The smallest basis set has been taken from the work by Laerdahl and Schwerdtfeger, ${ }^{87}$ who considered several basis sets in their work. From these we have chosen the basis type1, which is slightly better than double zeta quality. Then we systematically improved the size by using the Dyall-type all-electron basis sets, that is, dyall.ae $3 z$ and dyall.ae $4 z^{108}$ (a higher cardinal number is presently not available). We will employ the optimal cardinal number obtained form this study to the other molecules of this series. The Gaussian model of nuclei has been employed for all the molecules in this series. ${ }^{30}$ We have chosen an approximate version of the DC Hamiltonian, with the $(S S \mid S S)$ class of integrals neglected. Our choice of correlation space consists of all the occupied orbitals, but the virtual orbital space has been truncated by choosing an energy threshold of $100 E_{h}$.

In Figure $1 E_{P V}$ is traced as a function of dihedral angle at both the HF and CCSD levels of theory, using the cc$p V D Z+3 p$ basis set ${ }^{87}$ to demonstrate the effect of correlation. The curve we obtained is of sinusoidal type, in agreement with previous studies. ${ }^{8,93-95,97,98,100}$ The effect of correlation gives a negative vertical shift to the $E_{P V}$ value throughout the

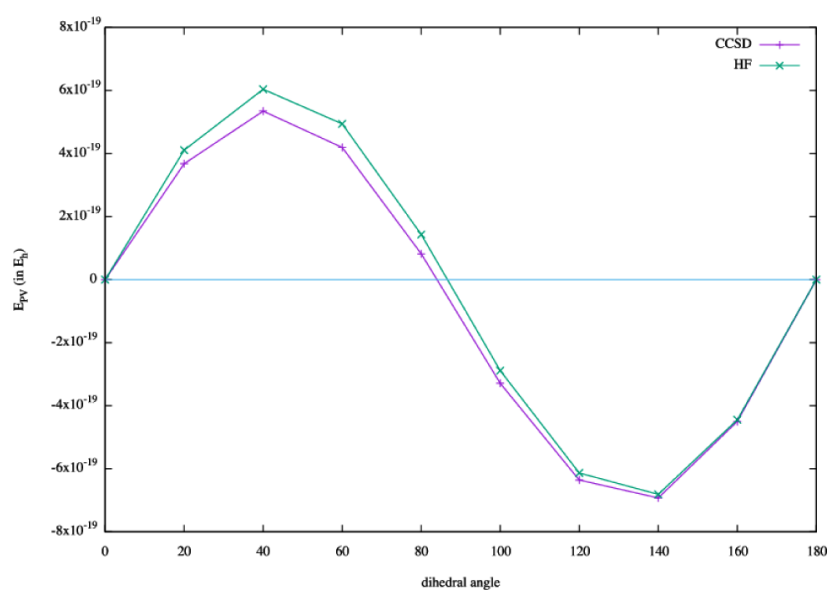

FIG. 1. Total parity violating energy $E_{P V}$ as a function of torsional angle curve for $\mathrm{H}_{2} \mathrm{O}_{2}$ at the $\mathrm{HF}$ and CCSD level of theory using the cc-pVDZ $+3 \mathrm{p}$ basis set. ${ }^{87}$ 
TABLE I. Parity violating energies $E_{P V}$ for P enantiomers of $H_{2} X_{2}$ molecules at various levels of theory. We have considered $45^{\circ}$ torsional angle for all the molecules. Finite-field results (orbital-relaxed) are marked as ff; all other numbers are analytical.

\begin{tabular}{|c|c|c|c|c|}
\hline Molecule & Hamiltonian & Method & Basis set for $\mathrm{X}$ & $E_{P V} / 10^{-20} E_{h}$ \\
\hline \multirow[t]{9}{*}{$\mathrm{H}_{2} \mathrm{O}_{2}$} & \multirow[t]{8}{*}{ DC } & \multirow[t]{3}{*}{$\mathrm{CCSD}^{\mathrm{a}}$} & cc-pVDZ+3p & -61.26 \\
\hline & & & dyall.ae3z & -53.23 \\
\hline & & & dyall.ae $4 \mathrm{z}$ & -55.83 \\
\hline & & \multirow[t]{3}{*}{$\mathrm{HF}^{\mathrm{a}}$} & $c c-p V D Z+3 p$ & -67.90 \\
\hline & & & dyall.ae $3 z$ & -60.51 \\
\hline & & & dyall.ae4z & -63.76 \\
\hline & & $\operatorname{CCSD}(f f)^{b}$ & $c c-p V D Z+3 p$ & -61.80 \\
\hline & & $\mathrm{MP} 2^{\mathrm{c}}$ & $c c-p V D Z+3 p$ & -57.88 \\
\hline & NR & $\operatorname{CCSD}^{\mathrm{d}}$ & cc-pVQZ & -51.69 \\
\hline \multirow[t]{7}{*}{$\mathrm{H}_{2} \mathrm{~S}_{2}$} & \multirow[t]{6}{*}{ DC } & \multirow[t]{2}{*}{$\mathrm{CCSD}^{\mathrm{a}}$} & cc-pCVTZ & -1821.03 \\
\hline & & & $c c-p V D Z+2 p$ & -2088.76 \\
\hline & & \multirow[t]{2}{*}{$\mathrm{HF}^{\mathrm{a}}$} & cc-pCVTZ & -1825.86 \\
\hline & & & $c c-p V D Z+2 p$ & -2078.20 \\
\hline & & $\operatorname{CCSD}(\mathrm{ff})^{\mathrm{b}}$ & $c c-p V D Z+2 p^{e}$ & -2165.23 \\
\hline & & $\mathrm{MP} 2^{\mathrm{c}}$ & $c c-p V D Z+2 p$ & -2112.0 \\
\hline & NR & $\operatorname{CCSD}^{\mathrm{d}}$ & cc-pVQZ & -2248.6 \\
\hline
\end{tabular}

a This study.

${ }^{\mathrm{b}}$ Thyssen et al. ${ }^{83}$

${ }^{\mathrm{c}}$ van Stralen et al. ${ }^{16}$

${ }^{\mathrm{d}}$ Horný and Quack. ${ }^{107}$

${ }^{\mathrm{e}}$ The authors report cc-pVDZ+3p, but this appears to be incorrect.

curve. We have obtained a maximum correlation contribution at $60^{\circ}$ torsional angle, and minimum is at $160^{\circ}$ torsional angle. Table I gives CCSD and HF values of $E_{P V}$ at $45^{\circ}$ torsional angle and indicates the convergence of $E_{P V}$ with respect to the basis set. Clearly, there is a significant difference in values when the double-zeta basis is compared with the triple-zeta one, whereas the effect of further increasing the cardinal number is quite small. We will prefer triple zeta quality basis set for our calculations on the heavier homologues of $\mathrm{H}_{2} \mathrm{O}_{2}$. We have also compared our findings with previous studies in Table I. Our analytical number matches very well the orbitalrelaxed finite-field numbers reported by Thyssen et al. ${ }^{83}$ when the basis set is exactly the same. ${ }^{109}$ The slight discrepancy in value may be attributed to orbital relaxation in the latter case, which is accordingly quite small. The difference with the MP2 value, when we use the same basis set, is not very large, as has been seen earlier. ${ }^{83}$

For the next candidate in this series, that is, $\mathrm{H}_{2} \mathrm{~S}_{2}$, we apply the cc-pCVTZ basis. Here we freeze the 1s2s orbitals of the sulfur atoms, as motivated by the MP2 study by van Stralen et al. ${ }^{16}$ For the virtual orbital space we again choose $100 E_{h}$ as the energy threshold. We vary the torsional angle from $0^{\circ}$ to $180^{\circ}$ with a fixed grid size of $15^{\circ}$. We observe the same sinusoidal behaviour of the $E_{P V}$ vs $\tau_{e}$ curve (Figure 2) as seen for $\mathrm{H}_{2} \mathrm{O}_{2}$. However, the effect of correlation is less dominant for this molecule, and correlation contributions have both negative and positive signs along the curve.

When we compare our cc-pCVTZ results at $45^{\circ}$ torsional angle for $\mathrm{H}_{2} \mathrm{~S}_{2}$ with previous findings in Table $\mathrm{I}$, we see a significant difference in values. In order to directly compare with the CCSD orbital-relaxed finite field number of Thyssen et al. ${ }^{83}$ we calculated the parity violating energy in the

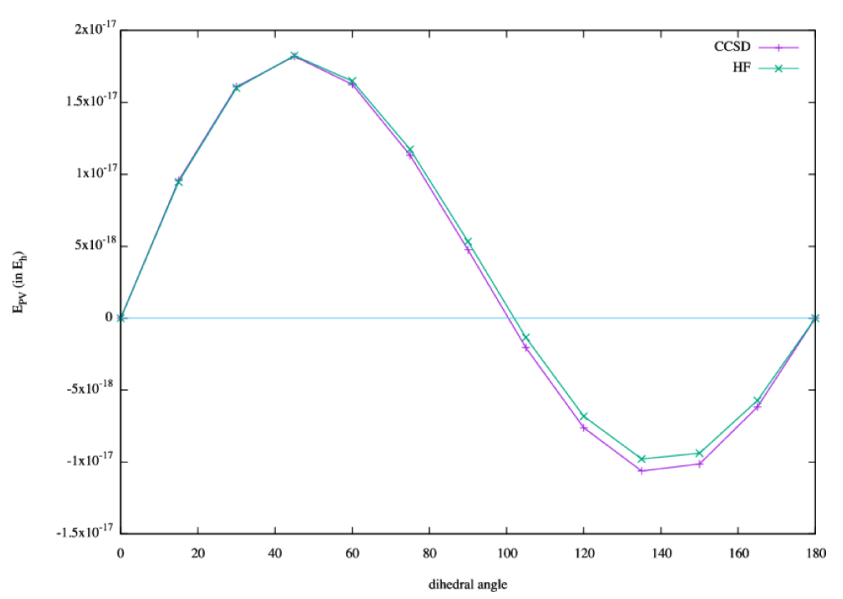

FIG. 2. Total parity violating energy $E_{P V}$ as a function of torsional angle curve for $\mathrm{H}_{2} \mathrm{~S}_{2}$ at the $\mathrm{HF}$ and CCSD level of theory using the cc-pCVTZ basis.

cc-pVDZ+2p basis with all electrons correlated. The discrepancy is now reduced to $76 \times 10^{-20} E_{h}$ (3.5\%), which is essentially due to orbital relaxation. At the orbitalunrelaxed MP2 level, switching off orbital relaxation in the implementation of van Stralen et al., ${ }^{16}$ we obtain $-2013.89 \times 10^{-20} E_{h}$, whereas using the orbital-relaxed MP2 code $^{16}$ the result is $-2113.83 \times 10^{-20} E_{h}$. The effect of orbital relaxation at the MP2 level is thus more important. The latter number agrees well with that reported by van Stralen et al., ${ }^{16}$ with the $1 \mathrm{~s} 2 \mathrm{~s} 2 \mathrm{p}$ orbitals of sulfur frozen.

In Table I we also included recent CCSD results reported by Horný and Quack. ${ }^{107}$ However, direct comparison with our values is less straightforward. Not only are basis sets and geometries different, but the parity-violating energy has been calculated as a linear response function by introducing a oneelectron spin-orbit operator with effective nuclear charge as a perturbation in an otherwise nonrelativistic calculation. We note, however, that with the triple zeta basis set we obtain very good agreement with the value reported for $\mathrm{H}_{2} \mathrm{O}_{2}$ by Horný and Quack, whereas a more significant discrepancy is observed for $\mathrm{H}_{2} \mathrm{~S}_{2}$, probably mostly due to scalar relativistic effects.

For $\mathrm{H}_{2} \mathrm{Se}_{2}$ and $\mathrm{H}_{2} \mathrm{Te}_{2}$ we have chosen $(n-1) \mathrm{d} n \mathrm{~s} n \mathrm{p}$ as the active occupied orbital space, where $n$ is the principal quantum number of the valence shell. The energy cutoff for the virtual orbitals was set to $40 E_{h}$. In Table II, we report our calculated $E_{P V}$ values at $45^{\circ}$ torsional angle for the complete series $\mathrm{H}_{2} \mathrm{X}_{2}(\mathrm{X}=\mathrm{O}, \mathrm{S}, \mathrm{Se}, \mathrm{Te})$ of molecules. An interesting observation is that the correlation contribution is on the order of $10 \%$ for $\mathrm{H}_{2} \mathrm{O}_{2}$, but is reduced by one order of magnitude or

TABLE II. Total parity violating energy shift (in $E_{h}$ ) at the CCSD level for the $\mathrm{H}_{2} \mathrm{X}_{2}(\mathrm{X}=\mathrm{O}, \mathrm{S}, \mathrm{Se}, \mathrm{Te})$ molecules at $45^{\circ}$ dihedral angle. Basis sets employed (both atoms): dyall.ae $3 \mathrm{z}\left(\mathrm{H}_{2} \mathrm{O}_{2}\right)$, cc-pCVTZ $\left(\mathrm{H}_{2} \mathrm{~S}_{2}\right)$ and dyall.cv $3 z$ $\left(\mathrm{H}_{2} \mathrm{Se}_{2}, \mathrm{H}_{2} \mathrm{Te}_{2}\right) . \Delta \mathrm{E}_{\boldsymbol{P V}}^{\text {corr }}$ corresponds to the energy shift (in $E_{h}$ ) at the CCSD level with respect to the HF method.

\begin{tabular}{lrrrr}
\hline \hline & \multicolumn{1}{c}{$\mathrm{H}_{2} \mathrm{O}_{2}$} & \multicolumn{1}{c}{$\mathrm{H}_{2} \mathrm{~S}_{2}$} & \multicolumn{1}{c}{$\mathrm{H}_{2} \mathrm{Se}_{2}$} & \multicolumn{1}{c}{$\mathrm{H}_{2} \mathrm{Te}_{2}$} \\
\hline $\mathrm{E}_{P V}$ & $-53.23 \times 10^{-20}$ & $-18.21 \times 10^{-18}$ & $-21.15 \times 10^{-16}$ & $-32.89 \times 10^{-15}$ \\
$\Delta \mathrm{E}_{P V}^{c o r r}$ & $7.4 \times 10^{-20}$ & $4.8 \times 10^{-20}$ & $2.4 \times 10^{-17}$ & $2.8 \times 10^{-16}$ \\
\hline \hline
\end{tabular}


more for the heavier homologues. There has been a previous analysis for that by Berger et al., ${ }^{101}$ where they have compared the ZORA-DFT numbers of theirs with the DC Hamiltonian based MP2 numbers by van Stralen et al. ${ }^{16}$ Their conclusion was that while MP2 numbers underestimate the correlation contribution of a DFT calculation, it overestimates that of a $\operatorname{CCSD}(\mathrm{T})$ calculation. Our numbers also corroborate with the overestimation of correlation by MP2, for which we have not carried out any calculation, but have observed the qualitative trend in the work by van Stralen et al. ${ }^{16}$ However, it should be pointed out that it is possible to provide a definite benchmark number only when an analytic $\operatorname{CCSD}(\mathrm{T})$ energy gradient implementation will be available.

\section{B. Electric field gradient}

We next consider the interplay between SOC and electron correlation by calculating the electric field gradient (EFG) at the ${ }^{209} \mathrm{Bi}$ nucleus (nuclear spin $\mathrm{I}=9 / 2$ ) in the $\mathrm{BiX}$ $(\mathrm{X}=\mathrm{N}, \mathrm{P})$ series of molecules, for which spin-orbit effects may be expected to be prominent. The corresponding nuclear quadrupole coupling constant (NQCC; in $\mathrm{MHz}$ ) may be expressed as

$$
\mathrm{NQCC}\left({ }^{209} \mathrm{BiX}\right)=234.9647 \times Q\left({ }^{209} \mathrm{Bi}\right) q_{z z}(\mathrm{Bi}),
$$

where $\mathrm{Q}$ is the nuclear quadrupole moment of ${ }^{209} \mathrm{Bi}$ in barns (b) and $q_{z z}$ is the electric field gradient (in atomic units $E_{h} / e a_{0}^{2}$ ) along the molecular axis at the position of the bismuth nucleus.

The basis set for $\mathrm{Bi}$ has been taken from the work by Teodoro and Haiduke, ${ }^{84}$ where they have incremented the Relativistic Adapted Gaussian Basis Set (RAGBS), previously developed by Haiduke and Da Silva. ${ }^{110}$ For the lighter atom in those molecules, that is, for $\mathrm{N}$ and $\mathrm{P}$, we have chosen the cc-pVTZ basis set. ${ }^{111,112}$ An approximate version of the DC Hamiltonian was used, where the $(S S \mid S S)$ class of integrals is neglected and a simple Coulombic correction added to the energy. ${ }^{113}$ To estimate the contribution from SOC, we have also employed the Spin-Free (SF)-DC Hamiltonian ${ }^{23,114}$ which allows us to define the SOC contribution as the difference between the results obtained with the full DC Hamiltonian and with the SF Hamiltonian. The correlating orbital space has been restricted by selecting all the orbitals between -6 and $20 E_{h}$, following the suggestion by Teodoro and Haiduke. ${ }^{84}$

We have summarized our results in Table III. For the BiN molecule at the SF level, electron correlation gives a $16.31 \%$ negative shift to the absolute value of $q_{z z}$. At the DC level, on the other hand, we get a huge positive shift of $57.2 \%$ to the absolute value of $q_{z z}$. This immediately shows that the SOC and correlation cannot be treated independently and that both are important to obtain a quantitatively correct total EFG value. With further analysis we can see that SOC reduces the absolute value of $q_{z z}$ by $7.01 E_{h} / e a_{0}^{2}$ at the mean-field level, in line with other previous studies of different molecules. ${ }^{117,118}$ The extent of coupling between SOC and correlation can be singled out by subtracting the mean field $\triangle S O C$ contribution from that at the CCSD level, which is a significantly large value $-5.62 E_{h} / e a_{0}^{2}$. For $\mathrm{BiP}$ the role of SOC is less dramatic,
TABLE III. Calculated electric field gradient $q_{z z}$ at the Bi nucleus of the $\mathrm{BiN}$ and BiP molecules. Only electronic contributions are given in the table. The nuclear contribution is 0.2864 and $0.3685 E_{h} / e a_{0}^{2}$ for $\mathrm{BiN}$ and $\mathrm{BiP}$, respectively. Bond lengths for $\mathrm{BiN}$ and $\mathrm{BiP}$ are chosen as $1.9349 \AA^{115}$ and $2.2934 \AA,{ }^{116}$ respectively. We used a $31 \mathrm{~s} 24 \mathrm{p} 18 \mathrm{~d} 12 \mathrm{f} 2 \mathrm{~g}$ basis set for Bi and cc-pVTZ for $\mathrm{N}$ and P. $\triangle S O C$ refers to the spin-orbit coupling contribution at the indicated level of theory.

\begin{tabular}{|c|c|c|c|c|c|}
\hline Molecule & Hamiltonian & Method & $\begin{array}{c}q_{z z} \\
\text { (in } E_{h} / e a_{0}^{2} \text { ) }\end{array}$ & $\%$ correlation & $\triangle S O C$ \\
\hline \multirow[t]{4}{*}{$\mathrm{BiN}$} & \multirow[t]{2}{*}{ SF-DC } & $\mathrm{HF}$ & -13.0961 & -16.31 & 7.0121 \\
\hline & & CCSD & -10.9596 & $\ldots$ & 1.3958 \\
\hline & \multirow[t]{2}{*}{$\mathrm{DC}$} & $\mathrm{HF}$ & -6.0840 & +57.20 & $\ldots$ \\
\hline & & CCSD & -9.5638 & $\cdots$ & $\ldots$ \\
\hline \multirow[t]{4}{*}{$\mathrm{BiP}$} & \multirow[t]{2}{*}{ SF-DC } & $\mathrm{HF}$ & -13.6345 & -21.06 & 3.5969 \\
\hline & & CCSD & -10.7626 & $\ldots$ & 1.1318 \\
\hline & \multirow[t]{2}{*}{$\mathrm{DC}$} & $\mathrm{HF}$ & -10.0376 & -4.05 & $\cdots$ \\
\hline & & CCSD & -9.6308 & $\ldots$ & $\ldots$ \\
\hline
\end{tabular}

and our simple analysis tells us that the role of coupling between correlation and SOC is $2.47 E_{h} / e a_{0}^{2}$ in that case.

If we combine our DC-CCSD EFG value with the NQCC data from Cooke et al. ${ }^{84,116}$ we obtain a nuclear quadrupole moment of $-415.1 \mathrm{mb}$ for ${ }^{209} \mathrm{Bi}$, in line with Teodoro and Haiduke (CCSD: $-420(8) \mathrm{mb}){ }^{84}$ This clearly indicates that the present literature value of $-516(15) \mathrm{mb}^{119,120}$ needs revision, in particular since the triples contribution to the EFG is rather modest, on the order of $5 \mathrm{mb} .{ }^{84}$ One further interesting point is that if we compare our orbital unrelaxed analytical NQM value with the relaxed numerical value by Teodoro and Haiduke, ${ }^{84}$ we see a difference of $5 \mathrm{mb}$. Therefore, for this particular case the effect of orbital relaxation is minor.

\section{SUMMARY AND FUTURE OUTLOOK}

In this article we have reported the first formulation and implementation of 4-component DC Hamiltonian based coupled cluster analytic derivative technique. The present implementation allows first-order energy derivative calculations of one-electron properties. We have used full permutational and double group symmetry of the integrals in the coupled cluster contraction section. This method has been applied to the calculation of zZ-component of EFG tensors at the $\mathrm{Bi}$ centre of $\mathrm{BiN}$ and $\mathrm{BiP}$ molecules. We have demonstrated a strong coupling between SOC and correlation for those molecules. In future work we plan to investigate the spin-orbit effect on the EFG in more detail. We have also calculated the total parity violating energy contribution for $\mathrm{H}_{2} \mathrm{X}_{2}(\mathrm{X}=\mathrm{O}, \mathrm{S}, \mathrm{Se}, \mathrm{Te})$ molecules and compared that with the previous studies. For the selected molecules the effect of electron correlation on the parity-violating energy is rather modest, but we would like to extend our benchmark study to more realistic candidate molecules where for instance the difference between HF and DFT results are more prominent (see for instance Ref. 82).

In the present implementation we have not included the $(\mathrm{T})$ correction to the calculated molecular properties. The main bottleneck of that implementation is the high 
memory requirements, which becomes particularly severe in the relativistic case due to the large prefactor caused by spin-orbit coupling. A viable implementation should include parallelization techniques. We are presently exploring suitable algorithms in that direction. In this implementation we have so far avoided the consideration of orbital relaxation via the Z-vector technique. For the calculation of geometrical derivatives and magnetic properties it is, however, mandatory to incorporate that contribution. The main bottleneck for the relativistic case arises because of the rotation between positive energy and negative energy orbitals, which will possibly require transformed negative energy MO integrals. Nevertheless, in view of the theoretical importance of that technique we are planning to implement it as well.

This work provides a generalized framework for tensor contraction in the relativistic single reference coupled cluster regime. We therefore plan to use this framework for the implementation of coupled cluster linear response. The response implementation will allow the calculation of excited state energies and higher-order molecular properties, most notably the NMR shielding tensor and indirect spin-spin coupling tensor.

An open-shell extension of this work will increase the scope of this work for magnetic properties such as the EPR g-tensor. In the spinor-based coupled cluster implementation one can calculate relaxed density matrix for simple open-shell systems. We are planning to use that benefit as outlined in Section III C.

\section{ACKNOWLEDGMENTS}

A.S. acknowledges his Ph.D. fellowship from the IndoFrench Centre for the Promotion of Advanced Research (IFCPAR) and would like to thank Debashis Mukherjee (Kolkata), Lan Cheng (John Hopkins), Andre Gomes (Lille), and Sangita Sen (Oslo) for many fruitful discussions. A.S. would also like to acknowledge computing time on the Linux clusters of Laboratoire de Chimie et Physique Quantiques (LCPQ, Toulouse) and Laboratoire de Physique des Lasers, Atomes et Molécules (PhLAM, Lille).

\section{APPENDIX A: WORKING EQUATIONS FOR $\Lambda$ EQUATIONS, INTERMEDIATES, AND DENSITY MATRICES}

The $\Lambda$ amplitudes have been expressed in terms of a limited number of intermediates, where we have largely followed the work of Gauss et al. ${ }^{121}$ The $\Lambda_{1}$ amplitude equation is given by

$$
\begin{aligned}
0= & \bar{F}_{a}^{i}+\sum_{e} \lambda_{e}^{i} \bar{F}_{a}^{e}-\sum_{m} \lambda_{a}^{m} \bar{F}_{m}^{i}+\sum_{m, e<f} \lambda_{e f}^{i m} * W_{a m}^{e f} \\
& -\sum_{e, f} G_{e}^{f} * W_{f a}^{e i}-\sum_{m n} G_{m}^{n} * W_{n a}^{m i} \\
& +\sum_{m e} \lambda_{e}^{m} * W_{a m}^{i e}-\sum_{m>n, e} \lambda_{a e}^{m n} * W_{m n}^{i e},
\end{aligned}
$$

whereas the $\Lambda_{2}$-equation is

$$
\begin{aligned}
0= & V_{a b}^{i j}+P(a b) \sum_{e} \lambda_{a e}^{i j} * \bar{F}_{b}^{e}-P(i j) \sum_{m} \lambda_{a b}^{i m} * \bar{F}_{m}^{j}+\sum_{m>n} \lambda_{a b}^{m n} * W_{m n}^{i j}+P(i j) p(a b) \sum_{m e} \lambda_{a e}^{i m} * W_{b m}^{j e} \\
& +P(a b) \sum_{e} V_{a e}^{i j} * G_{b}^{e}-\sum_{m} \lambda_{a}^{m} * W_{m b}^{i j}-P(i j) \sum_{m} V_{a b}^{i m} * G_{m}^{j}+P(i j) \sum_{m, e} V_{a b}^{m j} *\left(\lambda_{e}^{i} * t_{m}^{e}\right) \\
& +P(i j) \sum_{e} \lambda_{e}^{i} * V_{a b}^{e j}+P(i j) P(a b) \lambda_{a}^{i} \bar{F}_{b}^{j}+\sum_{e>f} \lambda_{e f}^{i j} * W_{a b}^{e f},
\end{aligned}
$$

where the permutation operator

$$
P(p q) f(p, q)=f(p, q)-f(q, p),
$$

appears. Most of the intermediates are constructed from the known Hamiltonian matrix elements and the already solved $\mathrm{T}$ amplitudes; we will hence call them fixed intermediates. The one-body fixed intermediates are

$$
\begin{aligned}
& \bar{F}_{m}^{i}=f_{m}^{i}+\sum_{e} f_{e}^{i} * t_{m}^{e}+\sum_{e n} V_{m e}^{i n} * t_{n}^{e}+\sum_{n, e>f} V_{e f}^{i n} * \tau_{m n}^{e f}, \\
& \bar{F}_{a}^{e}=f_{a}^{e}-\sum_{m} f_{a}^{m} * t_{m}^{e}-\sum_{m f} V_{f a}^{m e} * t_{m}^{f}-\sum_{m>n, f} V_{a f}^{m n} * \tau_{m n}^{e f}, \\
& \bar{F}_{e}^{m}=f_{e}^{m}+\sum_{n f} v_{e f}^{m n} * t_{n}^{f},
\end{aligned}
$$

whereas the two-body fixed intermediates are

$$
\begin{aligned}
W_{m n}^{i j}=V_{m n}^{i j}+ & P(m n) \sum_{e} V_{e n}^{i j} * t_{m}^{e}+\frac{1}{2} \sum_{e>f} V_{e f}^{i j} * \tau_{m n}^{e f}, \\
W_{e j}^{m b}= & V_{e j}^{m b}+\sum_{f} V_{e f}^{m b} * t_{j}^{f}-\sum_{n} V_{e j}^{m n} * t_{n}^{b} \\
& +\sum_{n f} V_{e f}^{m n} *\left(t_{j n}^{f b}-t_{j}^{f} t_{n}^{b}\right), \\
W_{m n}^{i e}= & V_{m n}^{i e}+\sum_{f} \bar{F}_{f}^{i} * t_{m n}^{e f}-\sum_{o} W_{m n}^{i o} * t_{o}^{e} \\
& +\sum_{f>g} V_{f g}^{i e} * \tau_{m n}^{f g}+P(m n) \sum_{f} \bar{W}_{f n}^{i e} * t_{m}^{f} \\
& +P(m n) \sum_{o f} V_{m f}^{i o} * t_{n o}^{e f},
\end{aligned}
$$




$$
\begin{gathered}
W_{a m}^{e f}=V_{a m}^{e f}+P(e f) \sum_{n g} V_{a g}^{e n} * \tau_{m n}^{g f}+\sum_{g} W_{a g}^{e f} * t_{m}^{g} \\
+\sum_{n} \bar{F}_{a}^{n} * t_{m n}^{e f}+\sum_{n>o} V_{a m}^{n o} * \tau_{n o}^{e f} \\
-P(e f) \sum_{n} \bar{W}_{a m}^{n f} * t_{n}^{e} \\
W_{a b}^{e f}=V_{a b}^{e f}-P(e f) \sum_{m} V_{a b}^{m f} * t_{m}^{e}+\sum_{m>n} V_{a b}^{m n} * \tau_{m n}^{e f} \\
\bar{W}_{e j}^{m b}=V_{e j}^{m b}-\sum_{n f} V_{e f}^{m n} * t_{n j}^{b f} \\
W_{i e}^{m n}=V_{i e}^{m n}+\sum_{f} t_{i}^{f} * V_{f e}^{m n} \\
W_{e f}^{a m}=V_{e f}^{a m}-\sum_{n} V_{e f}^{n m} * t_{n}^{a}
\end{gathered}
$$

To avoid higher than two-body intermediates we furthermore define two intermediates of mixed $\Lambda$ and $T$ type,

$$
\begin{aligned}
G_{a}^{e} & =-\sum_{m>n, f} \lambda_{a f}^{m n} * t_{m n}^{e f}, \\
G_{m}^{i} & =\sum_{m, e>f} \lambda_{e f}^{i n} * t_{m n}^{e f} .
\end{aligned}
$$

The density matrices are evaluated by using the converged $\mathrm{T}$ and $\Lambda$ as follows:

$$
\begin{gathered}
\gamma_{i}^{\prime j}=-\sum_{m, e>f} t_{i m}^{e f} * \lambda_{e f}^{j m}-\sum_{e} t_{i}^{e} * \lambda_{e}^{j}, \\
\gamma_{b}^{\prime a}=\sum_{m>n, e} t_{m n}^{a e} * \lambda_{b e}^{m n}+\sum_{m} t_{m}^{a} * \lambda_{b}^{m}, \\
\gamma_{i}^{\prime a}=t_{i}^{a}+\sum_{m e} \lambda_{e}^{m}\left(t_{i m}^{a e}-t_{i}^{e} t_{m}^{a}\right) \\
-\sum_{m>n, e>f} \lambda_{e f}^{m n}\left(t_{i n}^{e f} * t_{m}^{a}+t_{i}^{e} * t_{m n}^{a f}\right), \\
\gamma_{a}^{\prime i}=\lambda_{a}^{i}, \\
\gamma_{p}^{q}=\frac{1}{2}\left(\gamma_{p}^{\prime q}+\gamma_{q}^{\prime p}\right) .
\end{gathered}
$$

\section{APPENDIX B: USE OF THE TENSOR CONTRACTION ROUTINES}

As an illustration of the use of our implemented tensor contraction routines we consider a representative term from the $\Lambda$-equation,

$$
\delta \lambda_{a b}^{i j}+P(i j) P(a b) \sum_{m e} \lambda_{a e}^{i m} * W_{b m}^{j e} \rightarrow \delta \lambda_{a b}^{i j} .
$$

In the above equation, we are contracting a rank-4 tensor $\lambda_{a e}^{i m}$ with a rank-4 tensor $W_{b m}^{j e}$ to generate another rank-4 tensor $\delta \lambda_{a b}^{i j}$. Therefore, it belongs to the contraction class 444 . In the code the corresponding subroutine is called as

cal1 contraction_444((/"o1", "o3", "p1", "p3"/), (/"o2", "p3", "p2", "o3"/), \&

\& (/"o1" , "o2" , "p1" , "p2"/) , ResL2, 1.0d0,1.0d0,nrep, RightTensor=w_voov, \&

\& LeftTensor=L2).

The first three arguments to the subroutine are orbital strings for the input and output tensors in the order they appear from a diagram. Indices are numbered with "o" and "p" referring to occupied (hole) and virtual (particle) orbitals, respectively. Then, we provide the name of the output tensor (or arrays), the diagram factor, the scaling factor of the product tensor, the number of irreducible representations (nrep), and finally the names of the input tensors (or arrays). In this example (Eq. (B1)) the input tensors would be reshaped to

$$
\begin{aligned}
\lambda(i, m, a, e) & \rightarrow \lambda^{\prime}(i, a, e, m), \\
W(j, e, b, m) & \rightarrow W^{\prime}(j, b, e, m) .
\end{aligned}
$$

Thereafter the BLAS matrix-matrix multiplication routine is employed to the tensors mapped onto matrices, e.g.,

$$
\lambda^{\prime}(i a, e m) * W^{\prime T}(e m, j b)=\delta \lambda^{\prime}(i a, j b) \rightarrow \delta \lambda(i, j, a, b),
$$

followed by a reshaping (sort) of the resultant tensor, as above. When explicit anti-symmetrization of the final tensors is needed (as it is in the present example), we first generate the full product tensor, and then perform the explicit antisymmetrization and pack them in triangular fashion. Again, this is also implicit in the placement of the orbital indices.

\section{APPENDIX C: USE OF DOUBLE GROUP SYMMETRY IN TENSOR CONTRACTION}

The DPD scheme is applicable to all possible contractions in coupled cluster theory and entails nested loops over irreps to skip contributions that are zero by symmetry. A particular challenge in the relativistic framework is that the irrep of the complex conjugate of a quantity, e.g., an orbital appearing in the bra position of an two-electron integral, is typically not equal to the irrep of the original quantity. This is symbolically indicated by $\Gamma_{p} \neq \Gamma_{p}^{*}$ and may occur for both fermion and boson irreps. An example are the irreps of the groups $C_{n}^{*}(n>1)$.

Using the equation for generic tensor contraction (Equation (22)), we get

$$
\begin{aligned}
A\left(\left(\Gamma_{k^{\prime}}^{*} \otimes \Gamma_{l^{\prime}}^{*} \ldots\right) \otimes\left(\Gamma_{i} \otimes \Gamma_{j} . .\right)\right. & \left.\equiv \Gamma_{0}\right) B\left(\left(\Gamma_{i^{\prime}}^{*} \otimes \Gamma_{j^{\prime}}^{*}\right) \otimes\left(\Gamma_{k} \otimes \Gamma_{l} . .\right) \equiv \Gamma_{0}\right) \\
& =C\left(\left(\Gamma_{K_{f}^{\prime}}^{*} \otimes \Gamma_{I_{f}^{\prime}}^{*}\right) \otimes\left(\Gamma_{I_{f}} \otimes \Gamma_{K_{f}}\right) \equiv \Gamma_{0}\right)
\end{aligned}
$$


when the explicit symmetry label of each index is considered. In that equation $K_{f}^{\prime}$ represents a set of uncontracted or free indices coming from the $\left(k^{\prime}, l^{\prime} ..\right)$ orbital set and $\Gamma_{K_{f}^{\prime}}^{*}$ is the direct product of all these indices. Likewise, $I_{f}^{\prime}$ stands for all the free indices from the $\left(i^{\prime}, j^{\prime} ..\right)$ set, $I_{f}$ is for the $(i, j .$.$) set,$ and $K_{f}$ is for the $(k, l .$.$) set. \Gamma_{0}$ is the totally symmetric irrep. The remaining orbitals which do not belong to the sets $K_{f}^{\prime}$, $I_{f}^{\prime}, I_{f}$, and $K_{f}$ are contracted, and the direct product irreps of them within A and B are the same.

As we have already mentioned, every contraction is mapped onto Equation (23). For this case the symmetry restrictions entails that

$$
\begin{aligned}
& A \rightarrow A^{\prime}:\left(\Gamma_{K_{f}^{\prime}}^{*} \otimes \Gamma_{I_{f}}\right) \otimes\left(\Gamma_{K_{c}^{\prime}}^{*} \otimes \Gamma_{I_{c}}\right)=\Gamma_{f_{1}}^{\prime} \otimes \Gamma_{c_{1}}^{\prime}=\Gamma_{0}, \\
& B \rightarrow B^{\prime}:\left(\Gamma_{I_{c}^{\prime}}^{*} \otimes \Gamma_{K_{c}}\right) \otimes\left(\Gamma_{I_{f}^{\prime}}^{*} \otimes \Gamma_{K_{f}}\right)=\Gamma_{c_{2}}^{\prime} \otimes \Gamma_{f_{2}}^{\prime}=\Gamma_{0}, \\
& C \leftarrow C^{\prime}:\left(\Gamma_{K_{f}^{\prime}}^{*} \otimes \Gamma_{I_{f}}\right) \otimes\left(\Gamma_{I_{f}^{\prime}}^{*} \otimes \Gamma_{K_{f}}\right)=\Gamma_{f_{1}}^{\prime} \otimes \Gamma_{f_{2}}^{\prime}=\Gamma_{0},
\end{aligned}
$$

where the subscript " $\mathrm{f}$ " stands for free indices and subscript " $\mathrm{c}$ " stands for contractable indices. Equations (C2)-(C4) define the symmetry structure of the tensors after employing the sorting step. We have introduced a new symmetry label $\Gamma^{\prime}$ to indicate that the sorted indices can originate from the ket (that is, $\Gamma$ ) or bra (that is, $\Gamma^{*}$ ) position or from both of them. Consequently, multiplication tables have been set up for the direct products $\left(\Gamma_{p} \otimes \Gamma_{q}\right)$ and $\left(\Gamma_{p} \otimes \Gamma_{q}^{*}\right)$, where $\Gamma_{p}$ and $\Gamma_{q}$ may refer to both fermion and boson irreps.

Now, with a specific example as in Equation (B1), the input tensor $\lambda$ appears as an array $\left(i, m, a, e, \Gamma_{a e}\right)$ containing only elements for which

$$
\left(\Gamma_{i}^{*} \otimes \Gamma_{m}^{*}\right) \otimes\left(\Gamma_{a} \otimes \Gamma_{e}\right)=\Gamma_{i m}^{*} \otimes \Gamma_{a e}=\Gamma_{0},
$$

where $\Gamma_{0}$ is the totally symmetric irrep. Upon sorting it will be reshaped to an array $\left(i, a, e, m, \Gamma_{e m}^{\prime}\right)$, where $\Gamma_{e m}^{\prime}=\Gamma_{e} \otimes \Gamma_{m}^{*}$. The arrays $W$ and $\delta \lambda$ will undergo similar treatment.

Finally, one contraction with explicit symmetry labelling can be written as

$$
A^{\prime}\left(\Gamma_{f_{1}}^{\prime}, \Gamma_{c_{1}}^{\prime}\right) \otimes B^{\prime}\left(\Gamma_{c_{2}}^{\prime}, \Gamma_{f_{2}}^{\prime}\right)=C^{\prime}\left(\Gamma_{f_{1}}^{\prime}, \Gamma_{f_{2}}^{\prime}\right) .
$$

In this step we make sure that $\Gamma_{c_{1}}^{\prime}=\Gamma_{c_{2}}^{\prime}$ and all other symmetry restrictions follow from Equations (C2)-(C4). In our specific example, we multiply $\lambda$ and $W$ in the matrix form $\lambda\left(i a, e m: \Gamma_{e m}^{\prime}\right)$ and $W\left(j b, e m: \Gamma_{e m}^{\prime}\right)$ by using a BLAS matrix-matrix multiplication routine. Notice that we need to transpose the array $\mathrm{W}$ for carrying out the multiplication. Finally the product array $\delta \lambda\left(i a: \Gamma_{i a}^{\prime}, j b: \Gamma_{j b}^{\prime}\right)$ is reshaped to $\delta \lambda(i, j, a, b)$, where $\Gamma_{i j}^{*}=\Gamma_{a b}$.

These sorting steps are at most of order $N^{4}$ (with $N$ the number of orbitals) and should be insignificant relative to the $N^{6}$ order contractions. Due to the higher speed of the latter it is in practice, however, necessary to also consider optimization of these sorting steps as they often require non-contiguous memory access.

\footnotetext{
${ }^{1}$ M. Gell-Mann, Il Nuovo Cimento 4, 848 (1956).

${ }^{2}$ K. Schwarzschild, Gött. Nach., Math.-Phys. Kl. 126 (1903).

${ }^{3}$ T. Saue, Adv. Quantum Chem. 48, 383 (2005).

${ }^{4}$ T. Saue, ChemPhysChem 12, 3077 (2011).

${ }^{5}$ H. Bolvin, ChemPhysChem 7, 1575 (2006).
}

${ }^{6} \mathrm{R}$. Berger, "Parity-violation effects in molecules," in Relativistic Electronic Structure Theory, Part 2, Applications, edited by P. Schwerdtfeger (Elsevier, Netherlands, 2004), pp. 188-288.

${ }^{7}$ M. Quack, J. Stohner, and M. Willeke, Annu. Rev. Phys. Chem. 59, 741 (2008).

${ }^{8}$ R. Bast, A. Koers, A. S. P. Gomes, M. Ilias, L. Visscher, P. Schwerdtfeger, and T. Saue, Phys. Chem. Chem. Phys. 13, 864 (2011).

${ }^{9}$ Y. Nomura, Y. Takeuchi, and N. Nakagawa, Tetrahedron Lett. 10, 639 (1969).

${ }^{10}$ I. Morishima, K. Endo, and T. Yonezawa, J. Chem. Phys. 59, 3356 (1973).

${ }^{11}$ L. Visscher, T. Enevoldsen, T. Saue, and J. Oddershede, J. Chem. Phys. 109, 9677 (1998).

${ }^{12} \mathrm{~F}$. Aquino, N. Govind, and J. Autschbach, J. Chem. Theory Comput. 6, 2669 (2010).

${ }^{13}$ S. Knecht, S. Fux, R. van Meer, L. Visscher, M. Reiher, and T. Saue, Theor. Chem. Acc. 129, 631 (2011).

${ }^{14}$ P. O. Lipas, P. Pyykkö, and E. Pajanne, J. Chem. Phys. 58, 3248 (1973).

${ }^{15}$ P. Pyykkö, Chem. Rev. 88, 563 (1988).

${ }^{16}$ J. N. van Stralen, L. Visscher, C. V. Larsen, and H. J. A. Jensen, Chem. Phys. 311, 81 (2005).

${ }^{17}$ I. Kim, Y. C. Park, H. Kim, and Y. S. Lee, Chem. Phys. 395, 115 (2012).

${ }^{18}$ F. Wang, J. Gauss, and C. van Wüllen, J. Chem. Phys. 129, 064113 (2008).

${ }^{19}$ F. Wang and J. Gauss, J. Chem. Phys. 129, 174110 (2008).

${ }^{20}$ L. Cheng and J. Gauss, J. Chem. Phys. 134, 244112 (2011).

${ }^{21}$ L. Cheng and J. Gauss, J. Chem. Phys. 135, 084114 (2011).

${ }^{22}$ L. Cheng and J. Gauss, J. Chem. Phys. 135, 244104 (2011).

${ }^{23}$ L. Visscher and T. Saue, J. Chem. Phys. 113, 3996 (2000).

${ }^{24}$ S. Knecht and T. Saue, Chem. Phys. 401, 103 (2012).

${ }^{25}$ I. S. Lim and P. Schwerdtfeger, Phys. Rev. A 70, 062501 (2004).

${ }^{26}$ DIRAC, a relativistic $a b$ initio electronic structure program, Release DIRAC15 (2015), written by R. Bast, T. Saue, L. Visscher, and H. J. Aa. Jensen, with contributions from V. Bakken, K. G. Dyall, S. Dubillard, U. Ekstroem, E. Eliav, T. Enevoldsen, E. Fasshauer, T. Fleig, O. Fossgaard, A. S. P. Gomes, T. Helgaker, J. Henriksson, M. Ilias, Ch. R. Jacob, S. Knecht, S. Komorovsky, O. Kullie, J. K. Laerdahl, C. V. Larsen, Y. S. Lee, H. S. Nataraj, M. K. Nayak, P. Norman, G. Olejniczak, J. Olsen, Y. C. Park, J. K. Pedersen, M. Pernpointner, R. Di Remigio, K. Ruud, P. Salek, B. Schimmelpfennig, J. Sikkema, A. J. Thorvaldsen, J. Thyssen, J. van Stralen, S. Villaume, O. Visser, T. Winther, and S. Yamamoto, see http://www.diracprogram.org.

${ }^{27}$ G. E. Brown and D. G. Ravenhall, Proc. R. Soc. A 208, 552 (1951).

${ }^{28}$ J. Sucher, Phys. Rev. A 22, 348 (1980).

${ }^{29}$ A. Almoukhalalati, S. Knecht, H. J. A. Jensen, K. G. Dyall, and T. Saue, J. Chem. Phys. 145, 074104 (2016).

${ }^{30}$ L. Visscher and K. G. Dyall, At. Data Nucl. Data Tables 67, 2007 (1997).

${ }^{31}$ J. Olsen and P. Jørgensen, J. Chem. Phys. 82, 3235 (1985).

${ }^{32}$ O. Christiansen, P. Jørgensen, and C. Hättig, Int. J. Quantum Chem. 68, 1 (1998).

${ }^{33}$ J. Gauss, in Modern Methods and Algorithms of Quantum Chemistry, edited by J. Grotendorst (John von Neumann-Institute for Computing, Jülich, 2000), see http://webarchiv.fz-juelich.de/nic-series//Volume1/ Volume1.html.

${ }^{34}$ T. Saue, in Relativistic Electronic Structure Theory. Part 1. Fundamentals, edited by P. Schwerdtfeger (Elsevier, Amsterdam, 2002), p. 332.

${ }^{35} \mathrm{~T}$. Helgaker, S. Coriani, P. Jørgensen, K. Kristensen, J. Olsen, and K. Ruud, Chem. Rev. 112, 543 (2012).

${ }^{36}$ E. A. Hylleraas, Z. Phys. 65, 209 (1930).

${ }^{37}$ E. Wigner, Math. Natur. Anz. (Budapest) 53, 477 (1935).

${ }^{38}$ H. Koch, H. J. A. Jensen, P. Jørgensen, T. Helgaker, G. E. Scuseria, and H. F. Schaefer, J. Chem. Phys. 92, 4924 (1990).

${ }^{39}$ T. Helgaker and P. Jørgensen, Theor. Chim. Acta 75, 111 (1989).

${ }^{40}$ P. Jørgensen and T. Helgaker, J. Chem. Phys. 89, 1560 (1988).

${ }^{41}$ T. Helgaker and P. Jørgensen, in Methods in Computational Molecular Physics, edited by S. Wilson and G. H. F. Diercksen (Plenum, New York, 1992), p. 353421.

${ }^{42}$ B. Levy, Chem. Phys. Lett. 4, 17 (1969).

${ }^{43}$ P. Jørgensen and J. Linderberg, Int. J. Quantum Chem. 4, 587 (1970).

${ }^{44}$ E. Dalgaard and P. Jørgensen, J. Chem. Phys. 69, 3833 (1978).

${ }^{45}$ T. Helgaker, P. Jørgensen, and J. Olsen, Molecular Electronic Structure Theory (John Wiley \& Sons, Ltd., Chichester, 2000).

${ }^{46}$ J. Talman, Phys. Rev. Lett. 57, 1091 (1986).

${ }^{47}$ T. Saue and L. Visscher, in Theoretical Chemistry and Physics of Heavy and Superheavy Elements, edited by S. Wilson and U. Kaldor (Kluwer, Dordrecht, 2003), p. 211. 
${ }^{48}$ L. S. Cederbaum, J. Schirmer, and H. D. Meyer, J. Phys. A: Math. Gen. 22, 2427 (1989).

${ }^{49}$ M. Ziolkowski, B. Jansik, P. Jørgensen, and J. Olsen, J. Chem. Phys. 131, 124112 (2009).

${ }^{50}$ T. Helgaker, P. Jørgensen, and N. C. Handy, Theor. Chim. Acta 76, 227 (1989).

${ }^{51}$ H. J. Aa. Jensen, K. G. Dyall, T. Saue, and K. Fægri, J. Chem. Phys. 104, 4083 (1996).

${ }^{52}$ J. Thyssen, T. Fleig, and H. J. A. Jensen, J. Chem. Phys. 129, 034109 (2008).

${ }^{53}$ F. London, J. Phys. Radium 8, 397 (1937).

${ }^{54}$ R. Ditchfield, Mol. Phys. 27, 789 (1974).

${ }^{55}$ A. E. Hansen and T. D. Bouman, J. Chem. Phys. 82, 5035 (1985).

${ }^{56}$ J. Olsen, K. L. Bak, K. Ruud, T. Helgaker, and P. Jørgensen, Theor. Chim. Acta 90, 421 (1995).

${ }^{57}$ E. A. Salter, H. Sekino, and R. J. Bartlett, J. Chem. Phys. 87, 502 (1987).

${ }^{58}$ M. M. Sternheim, Phys. Rev. 128, 676 (1962).

${ }^{59}$ G. Aucar, T. Saue, L. Visscher, and H. J. A. Jensen, J. Chem. Phys. 110, 6208 (1999).

${ }^{60}$ R. Kobayashi, H. Koch, P. Jørgensen, and T. J. Lee, Chem. Phys. Lett. 211, 94 (1993).

${ }^{61}$ O. Christiansen, C. Hättig, and J. Gauss, J. Chem. Phys. 109, 4745 (1998).

${ }^{62}$ A. A. Auer and J. Gauss, J. Chem. Phys. 115, 1619 (2001).

${ }^{63}$ T. D. Crawford and H. Schaefer, Rev. Comput. Chem. 14, 33 (2000).

${ }^{64}$ J. Arponen, Ann. Phys. 151, 311 (1983).

${ }^{65}$ J. F. Stanton and R. J. Bartlett, J. Chem. Phys. 98, 7029 (1993).

${ }^{66}$ E. A. Salter, G. W. Trucks, and R. J. Bartlett, J. Chem. Phys. 90, 1752 (1989).

${ }^{67}$ J. Goldstone, Proc. R. Soc. A 239, 267 (1957).

${ }^{68}$ J. Č́ižek, J. Chem. Phys. 45, 4256 (1966).

${ }^{69}$ I. Shavitt and R. J. Bartlett, Many-Body Methods in Chemistry and Physics: MBPT and Coupled-Cluster Theory (Cambridge University Press, 2009), p. 532.

${ }^{70}$ L. Visscher, K. G. Dyall, and T. J. Lee, Int. J. Quantum Chem., Quantum Chem. Symp. 29, 411 (1995).

${ }^{71}$ L. Visscher, T. J. Lee, and K. G. Dyall, J. Chem. Phys. 105, 8769 (1996).

${ }^{72}$ L. Visscher, E. Eliav, and U. Kaldor, J. Chem. Phys. 115, 9720 (2001).

${ }^{73}$ S. Hirata, J. Phys. Chem. A 107, 9887 (2003).

${ }^{74}$ E. Solomonik, D. Matthews, J. R. Hammond, J. F. Stanton, and J. Demmel, J. Parallel Distrib. Comput. 74, 3176 (2014).

${ }^{75}$ E. Deumens, V. F. Lotrich, A. Perera, M. J. Ponton, B. A. Sanders, and R. J. Bartlett, Wiley Interdiscip. Rev.: Comput. Mol. Sci. 1, 895 (2011).

${ }^{76}$ E. Epifanovsky, M. Wormit, T. Ku, A. Landau, D. Zuev, K. Khistyaev, P. Manohar, I. Kaliman, A. Dreuw, and A. I. Krylov, J. Comput. Chem. 34, 2293 (2013)

${ }^{77}$ J. A. Calvin and E. F. Valeev, TiledArray: A general-purpose scalable blocksparse tensor framework, https://github.com/valeevgroup/tiledarray.

${ }^{78}$ C. L. Lawson, R. J. Hanson, D. R. Kincaid, and F. T. Krogh, ACM Trans. Math. Software 5, 308 (1979).

${ }^{79}$ J. F. Stanton, J. Gauss, J. D. Watts, and R. J. Bartlett, J. Chem. Phys. 94, 4334 (1991)

${ }^{80}$ H. Bethe, Ann. Phys. 3, 133 (1929).

${ }^{81}$ T. Saue and H. J. A. Jensen, J. Chem. Phys. 111, 6211 (1999).

${ }^{82}$ F. D. Montigny, R. Bast, A. S. P. Gomes, G. Pilet, N. Vanthuyne, C. Roussel, L. Guy, P. Schwerdtfeger, T. Saue, and J. Crassous, Phys. Chem. Chem. Phys. 12, 8792 (2010).
${ }^{83}$ J. Thyssen, J. K. Laerdahl, and P. Schwerdtfeger, Phys. Rev. Lett. 85, 3105 (2000).

${ }^{84}$ T. Q. Teodoro and R. L. A. Haiduke, Phys. Rev. A 88, 052504 (2013).

${ }^{85}$ R. A. Hegstrom, D. W. Rein, and P. G. H. Sandars, J. Chem. Phys. 73, 2329 (1980).

${ }^{86}$ M. Quack, Angew. Chem., Int. Ed. 41, 4618 (2002).

${ }^{87}$ J. K. Laerdahl and P. Schwerdtfeger, Phys. Rev. A 60, 4439 (1999).

${ }^{88} \mathrm{P}$. Schwerdtfeger, in Computational Spectroscopy, edited by J. Grunenberg (Wiley, 2010), pp. 201-221.

${ }^{89}$ B. Darquié, C. Stoeffler, A. Shelkovnikov, C. Daussy, A. Amy-Klein, C. Chardonnet, S. Zrig, L. Guy, J. Crassous, P. Soulard, P. Asselin, T. R. Huet, P. Schwerdtfeger, R. Bast, and T. Saue, Chirality 22, 870 (2010).

${ }^{90}$ C. Medcraft, R. Wolf, and M. Schnell, Angew. Chem., Int. Ed. 53, 11656 (2014).

${ }^{91}$ J. Erler and M. Ramsey-Musolf, Prog. Part. Nucl. Phys. 54, 351 (2005).

${ }^{92}$ S. F. Mason and G. E. Tranter, Chem. Phys. Lett. 94, 34 (1983).

${ }^{93}$ S. Mason and G. Tranter, Mol. Phys. 53, 1091 (1984).

${ }^{94}$ P. Lazzeretti and R. Zanasi, Chem. Phys. Lett. 279, 349 (1997).

${ }^{95}$ A. Bakasov, T.-K. Ha, and M. Quack, J. Chem. Phys. 109, 7263 (1998).

${ }^{96}$ A. Bakasov and M. Quack, Chem. Phys. Lett. 303, 547 (1999).

${ }^{97}$ R. Berger and M. Quack, J. Chem. Phys. 112, 3148 (2000).

${ }^{98}$ A. C. Hennum, T. Helgaker, and W. Klopper, Chem. Phys. Lett. 354, 274 (2002).

${ }^{99}$ G. Laubender and R. Berger, ChemPhysChem 4, 395 (2003).

${ }^{100}$ A. Bakasov, R. Berger, T.-K. Ha, and M. Quack, Int. J. Quantum Chem. 99, 393 (2004).

${ }^{101}$ R. Berger and C. van Wüllen, J. Chem. Phys. 122, 134316 (2005).

${ }^{102}$ V. Weijo, P. Manninen, and J. Vaara, J. Chem. Phys. 123, 054501 (2005).

${ }^{103}$ R. Bast, P. Schwerdtfeger, and T. Saue, J. Chem. Phys. 125, 064504 (2006).

${ }^{104}$ G. Laubender and R. Berger, Phys. Rev. A 74, 032105 (2006).

${ }^{105}$ R. Berger, J. Chem. Phys. 129, 154105 (2008).

${ }^{106}$ S. Nahrwold and R. Berger, J. Chem. Phys. 130, 214101 (2009).

${ }^{107}$ Ľ. Horný and M. Quack, Mol. Phys. 113, 1768 (2015).

${ }^{108}$ K. G. Dyall, Theor. Chem. Acc. 135, 1 (2016).

${ }^{109}$ Thyssen et al. provided the $E_{P V}$ value for the $\mathrm{X}$ atoms. We have calculated the total $E_{P V}$ value by neglecting the contribution from $\mathrm{H}$-atom and employing the values for the constant factors $-Q_{W, n}$ and $G_{F}$ given in the text.

${ }^{110}$ R. L. A. Haiduke and A. B. F. Da Silva, J. Comput. Chem. 27, 1970 (2006).

${ }^{111}$ D. E. Woon and T. H. Dunning, J. Chem. Phys. 98, 1358 (1993).

${ }^{112}$ T. H. Dunning, J. Chem. Phys. 90, 1007 (1989).

${ }^{113}$ L. Visscher, Theor. Chem. Acc. 98, 68 (1997).

${ }^{114}$ K. G. Dyall, J. Chem. Phys. 100, 2118 (1994).

${ }^{115}$ R. Breidohr, O. Shestakov, K. Setzer, and E. Fink, J. Mol. Spectrosc. 172, 369 (1995).

${ }^{116}$ S. A. Cooke, J. M. Michaud, and M. C. Gerry, J. Mol. Struct. 695-696, 13 (2004), winnewisser Special Issue.

${ }^{117}$ P. Pyykkö and M. Seth, Theor. Chem. Acc. 96, 92 (1997).

${ }^{118}$ E. van Lenthe and E. Jan Baerends, J. Chem. Phys. 112, 8279 (2000).

${ }^{119}$ J. Bieroń and P. Pyykkö, Phys. Rev. Lett. 87, 133003 (2001).

${ }^{120}$ P. Pyykkö, Mol. Phys. 106, 1965 (2008).

${ }^{121}$ J. Gauss, J. F. Stanton, and R. J. Bartlett, J. Chem. Phys. 95, 2623 (1991). 